\title{
Análisis psicosocial del prejuicio hacia trabajadoras sexuales ${ }^{*}$
}

DOI: https://doi.org/10.18046/recs.i3o.2879

\author{
Psycho-social Analysis of Prejudice towards Sex Workers \\ Débora Imhoff ${ }^{* *}$ \\ Instituto de Investigaciones Psicológicas \\ (CONICET y Universidad Nacional de Córdoba) \\ Matías Dreizil**** \\ Universidad Nacional de Córdoba (Córdoba, Argentina)
}

Silvina Brussino ${ }^{* * * *}$

Instituto de Investigaciones Psicológicas

(CONICET y Universidad Nacional de Córdoba)

\footnotetext{
* Investigación inscrita en el proyecto "Cultura política y participación ciudadana en Argentina”. Período: 2016- 2017 (01.01.2016 a 31.12.2017). Con subsidio del SECYT-UNC categoría A (Resolución SECYT No 313/2016, Resolución HCS No 313/16). Directora: Dra. Brussino. Investigadores del equipo: Dra. Imhoff y Lic. Dreizik. Artículo de investigación recibido el 02.04.2018 y aceptado el 16.09.2019.

** Investigadora CONICET. Equipo de Psicología Política del Instituto de Investigaciones Psicológicas (CONICET y Universidad Nacional de Córdoba) (Argentina). Correo electrónico: dimhoff@conicet.gov.ar ORCID: http://orcid. org/oooo-0oo2-2276-1893

*** Investigador UNC. Equipo de Psicología Política del Instituto de Investigaciones Psicológicas (CONICET y Universidad Nacional de Córdoba) (Argentina). Correo electrónico: matiasdreizik@gmail.com ORCID: https://orcid. org/oooo-0oo2-1140-4225

**** Investigadora CONICET. Equipo de Psicología Política del Instituto de Investigaciones Psicológicas (CONICET y Universidad Nacional de Córdoba) (Argentina).Correo electrónico: silvina.brussino@unc.edu.ar ORCID: http://orcid. org/oooo-0oo2-1087-644X
} 


\section{Cómo citar/How to cite}

Imhoff, Débora; Dreizik, Matías; Brussino, Silvina (2020). Análisis psicosocial del prejuicio hacia trabajadoras sexuales. Revista CS, 30, 173-196. https://doi.org/10.18046/ recs.i30.2879 


\section{Resumen}

El estudio analizó las variables psicosociales (religión, orientación de dominancia social) y psicopolíticas (autoritarismo, ideología política) que colaboran en la predicción del prejuicio hacia trabajadoras sexuales (PTS). Investigación realizada con estudiantes universitarios(as) en dos fases: fase instrumental para la construcción y evaluación de una escala de PTS, y seguidamente un estudio ex post facto retrospectivo simple. Tras ratificar las cualidades psicométricas de la escala, se efectuó un análisis de regresión múltiple. En ambas dimensiones del PTS, fueron el autoritarismo y la oposición a la igualdad dos de las variables que más aportaron a la predicción. También la religión y el autoposicionamiento ideológico ingresaron como predictores.

PALABRAS CLAVE:

prostitución, trabajo sexual, prejuicio, psicología política

This study analyzed the psycho-social and psycho-political variables that contribute to predict prejudice towards sex workers (PSW). A two-phase quantitative study with university students was carried out: phase one included an instrumental research oriented to the construction and evaluation of a scale of PSW; phase two consisted of an ex post facto study with simple retrospective design. After ratifying the psychometric qualities of the constructed scale, a multiple regression analysis was performed. In both PSW dimensions, authoritarianism and opposition to equality were two of the variables that most contributed to the prediction. Also, religion and ideological self-placement were included in the models.

\section{KEYWORDS:}

Prostitution, Sex Work, Prejudice, Political Psychology 



\section{Introducción}

La problemática del trabajo sexual es un tema de centralidad creciente en la agenda sociopolítica latinoamericana, y fue incorporada a la agenda de la Comisión Económica para América Latina (CEPAL), en su Comité Especial sobre Población y Desarrollo, en Quito (2012). Al respecto, se señalan las condiciones de vulnerabilidad y exclusión que sufren las mujeres que se dedican al trabajo sexual o prostitución.

A su vez, se trata de un fenómeno no exento de tensiones y debates, incluso en lo que concierne a las formas de nombrar y reconocer la identidad de estas mujeres. Sobre este tema, Musto y Trajtenberg (2011:139) enfatizan que, en lo que concierne al trabajo sexual, "existen desacuerdos en torno a cómo conceptualizarlo, cuáles son sus principales causas, y su status moral y político", y proponen definirlo como "el intercambio de comportamientos de base sexual (coito, bailes eróticos, etc.) a cambio de un bien o servicio valorado por la contraparte". A su vez, según Pecheny y Petracci (2006), existen dos posturas: por un lado, se define a estas mujeres como "en situación de prostitución" (destacando su carácter de víctimas, hablando de explotación sexual), mientras por otro se procura desvictimizarlas, nombrándolas como "trabajadoras sexuales" (Juliano, 2005), vinculándolo incluso a la perspectiva marxista del trabajo (Roveres; Dreizik; Correa, 2007).

En el marco de este trabajo, nos situaremos en esta última vertiente. En esta línea, Juliano (2005) hace un análisis sobre la situación de las mujeres trabajadoras sexuales en Europa, donde el no reconocimiento de las condiciones de trabajo para sus actividades les impide gozar de derechos y desarrollar sus potencialidades. Así, enfatiza que, en general, suelen ser mujeres pobres, y afirma que la discriminación afecta de forma diferente según la clase social a que se pertenezca. También Fonseca-Hernández (2004) señala el sufrimiento, rechazo y discriminación social que vivencian mujeres migrantes latinoamericanas que se desempeñan como trabajadoras sexuales en dicho continente.

Ello señala la importancia de analizar los prejuicios que se articulan socialmente en torno a este colectivo, en tanto actitudes negativas que luego repercuten en conductas específicas hacia las mujeres trabajadoras sexuales. Desde la psicología social, se comprende al prejuicio como un tipo particular de actitud y, en tanto tal, posee elementos cognitivos, emocionales y conductuales. Se trata de una actitud generalmente negativa hacia las personas que pertenecen a un determinado grupo social, basada en el simple hecho de que forman parte del mencionado grupo (Etchezahar; Ungaretti; Rabbia, 2017). Es decir, no se efectúa una evaluación sobre la persona en su singularidad, sino que esta se vuelve receptora de la actitud prejuiciosa por el hecho de formar parte de un grupo determinado. En ese sentido, tal actitud 
tiene un carácter homogeneizador o generalizador sobre todos los individuos que conforman un colectivo social, en torno del cual se posee una evaluación negativa. Esta generalización se presenta no solo como errónea, sino también como inflexible (Allport, 1954; Baron; Byrne, 2005).

Dado que los prejuicios funcionan como esquemas cognitivos, "los individuos con prejuicios hacia grupos específicos tienden a procesar la información sobre estos grupos de manera diferente a la manera en que procesan la información de otros grupos" (Baron; Byrne, 2005: 218). En esa línea, por ejemplo, las informaciones que vienen a ratificar la perspectiva que una persona prejuiciosa posee respecto de un grupo social determinado (tomemos por caso, el colectivo de trabajadoras sexuales) reciben mayor atención o se procesan de forma más cuidadosa. A su vez, la activación de los prejuicios adquiere, generalmente, un carácter implícito, esto es, puede activarse de forma automática e influir en el comportamiento de las personas prejuiciosas aun cuando estas no sean conscientes de que poseen este tipo de actitudes o bien las nieguen. Ello da cuenta del carácter moldeador sobre el comportamiento que posee el prejuicio.

Por otra parte, debe señalarse que el prejuicio no necesariamente se expresa de forma hostil y manifiesta. Pettigrew y Meertens (1995) postulan que es posible distinguir entre expresiones manifiestas y sutiles de prejuicio. Al respecto, Baron y Byrne (2005: 219) afirman que "en muchos casos, quienes mantienen actitudes negativas hacia miembros de algunos grupos no manifiestan su prejuicio de forma directa. Leyes, presión social y miedo a represalias sirven para disuadir a las personas a que lleven sus visiones prejuiciadas a la práctica de manera explícita". Ello impacta en las expresiones manifiestas de prejuicio y en su paso al acto, a través de acciones concretas de discriminación; no obstante, no implica, necesariamente, una desaparición o disminución del prejuicio. Por ello, la noción de prejuicio sutil posee un potencial analítico innegable.

El prejuicio sutil reúne expresiones prejuiciosas indirectas, menos evidentes y explícitas, con menor carga de hostilidad, y distantes. Son formas "mejor adaptadas socialmente, que se infieren a partir de la defensa de los valores tradicionales del propio grupo, junto a la idea de que ciertos grupos no los estarían respetando" (Etchezahar et al., 2017: 214). Incluye manifestaciones en las cuales se exageran las diferencias culturales con el exogrupo, y en ocasiones se sostienen visiones que aparentan ser positivas, pero a las que subyace un proceso de subestimación, "peyorativización” y desvalorización del otro.

El prejuicio también en concebido en tanto originado en el proceso de categorización social, lo cual lleva a sostener que se trata de un mecanismo generalizado. Es decir, "los individuos prejuiciosos hacia un determinado grupo minoritario (e. g. 
homosexuales), suelen serlo también hacia otros (e. g. inmigrantes)" (Etchezahar et al., 2017: 219). Se trataría así de una tendencia de las personas a manifestar prejuicios hacia grupos que son identificados como diferentes al propio, más allá de las características específicas de estos grupos.

No obstante, otros desarrollos relativizan esta idea al afirmar que, si bien es un proceso generalizado, la actitud prejuiciosa no se dirige hacia cualquier grupo social, sino hacia aquellos que son percibidos de forma similar. Así, Sibley y Duckitt (2008) mostraron que era posible clasificar a los diversos grupos entre aquellos considerados peligrosos (en tanto atentarían contra los valores tradicionales del propio grupo, por ejemplo, criminales, vendedores/as de drogas), derogados (por ejemplo, personas discapacitadas, obesas) y disidentes (por ejemplo, feministas y prostitutas). De acuerdo con Etchezahar et al. (2017: 220) dentro de esta última categoría se incluirían "grupos que son percibidos como amenazantes de los valores tradicionales de la mayoría y que, a su vez, se manifiestan reclamando por sus derechos". Los resultados de Sibley y Duckitt (2008) señalan que las personas prejuiciosas suelen dirigir su actitud negativa hacia grupos de una misma categoría, pero no necesariamente hacia grupos de las otras categorías. Similares resultados fueron hallados en Argentina (Etchezahar; Ungaretti; Brussino, 2015).

Las trabajadoras sexuales, en tanto colectivo organizado, estarían incluidas en el grupo denominado "disidente" (aunque generalmente en los estudios sobre este tema se las nomine como "prostitutas" y no como "trabajadoras"). Esta inclusión fue ratificada por estudios efectuados en Argentina, en torno a diversos grupos sociales receptores de actitudes prejuiciosas, en un marco donde los análisis factoriales exploratorios y confirmatorios ratifican que las trabajadoras sexuales integran este grupo, junto a gais, lesbianas, travestis, personas a favor del aborto y feministas (Ungaretti; Etchezahar; Brussino, 2015).

Según diversos autores y autoras, la psicología social ha prestado mayor atención al estudio del prejuicio racial, en detrimento de investigaciones en torno al prejuicio hacia otros grupos sociales (Quiles del Castillo; Betancor-Rodríguez; Rodríguez-Torres; Rodríguez-Pérez; Coello-Martel, 2003). Entre estos últimos, destacamos a las trabajadoras sexuales, población menos estudiada en este tipo de indagaciones. Ello se ve reflejado en el hecho de que no se cuenta, hasta la actualidad, con una escala para medir de forma específica las diversas expresiones de prejuicio hacia este colectivo social. En esa línea pretendió aportar el presente estudio, a través de una primera investigación instrumental orientada a construir y analizar las propiedades psicométricas de una escala de prejuicio hacia trabajadoras sexuales.

Por otra parte, también interesó conocer cuáles son las variables psicosociales y psicopolíticas que aportan a la predicción del prejuicio hacia este grupo social. Al 
respecto, la literatura evidencia que diversas variables se vinculan con el prejuicio. Así, la orientación a la dominancia social (SDO, por sus siglas en inglés) y el autoritarismo del ala de derechas (RWA, por sus siglas en inglés) parecen ser dos variables clave para comprender el prejuicio, en tanto se relacionan de forma positiva con diversos tipos de prejuicios, por ejemplo: el sexual (Norton; Herek, 2013; Páez; Hevia; Pesci; Rabbia, 2015; Quiles del Castillo et al., 2003), hacia afroamericanos(as) (Altemeyer, 1998), hacia personas con SIDA (Cunningham; Dollinger; Satz; Rotter, 1991), y hacia personas pobres (Gatica; Martini; Dreizik; Imhoff, 2017), entre otros. En consonancia, Duckitt (2006) afirma que estas dos variables constituyen las bases sobre las que se asienta el prejuicio generalizado.

No obstante, un estudio llevado a cabo en Perú muestra diferencias en el aporte predictivo de estas dos variables sobre distintos tipos de prejuicios (Rottenbacher; Espinosa; Magallanes, 2011). Al respecto, se afirma que, si bien tanto RWA como SDO influyen sobre el prejuicio, lo hacen de forma diferencial, en función del grupo social de que se trate. Ello se debería a que cada uno de estos constructos remite a dos formas diferenciales de comprender el orden social. Así, las personas guiadas por altos niveles de autoritarismo creen en "un control social coercitivo, en la obediencia y respeto por la autoridad y en la conformidad con normas y valores tradicionales" (Feldman, 2003, como se citó en Ungaretti et al., 2015: 31). Por ello, se trataría de individuos más tendientes a dirigir sus actitudes prejuiciosas hacia grupos que se percibe que atentan contra las tradiciones del grupo de pertenencia (peligrosos) (Duckitt; Sibley, 2007; Ungaretti et al., 2015).

En contraposición, las personas que poseen niveles altos de orientación a la dominancia social miran al mundo social como una "jungla competitiva", en función de lo cual estructuran las relaciones intergrupales en términos de jerarquía y no de igualdad. En ese sentido, buscan que su grupo de pertenencia detente más poder, dominio y superioridad respecto de otros grupos. Por ende, es esperable que muestren más prejuicio hacia grupos considerados inferiores o con los cuales poseen una disputa por recursos o poder (Sibley; Duckitt, 2008).

Un caso particular lo constituye el prejuicio hacia grupos disidentes, entre los que hemos situado a la población de nuestro estudio, las trabajadoras sexuales. Ciertos estudios denotan que estos grupos son concebidos no solo como amenazantes del orden y la estabilidad social, y peligrosos por atentar contra las tradiciones, sino que también constituirían una competencia. Por ello, en lo que concierne al prejuicio hacia estos grupos, tanto RWA como SDO aparecen como variables predictivas (Duckitt; Wagner; Du Plessis; Birum, 2002). Estos hallazgos han sido ratificados en Argentina (Ungaretti et al., 2015). 
Además de la orientación de dominancia social y el autoritarismo del ala de derechas, se ha encontrado que las personas religiosas (adhieren a una religión) manifiestan niveles altos de prejuicio hacia distintos grupos sociales (Norton; Herek, 2013; Páez et al., 2015), constituyéndose esta en otra variable de interés para nuestro estudio. A su vez, la adhesión a una ideología política conservadora o de derecha también se relaciona con niveles altos de prejuicio hacia diferentes colectivos. Por ejemplo, estudios locales muestran que las personas de derecha suelen mostrar niveles más altos de prejuicio hacia personas pobres (Gatica et al., 2015) o inmigrantes (Civalero; Brussino, 2016).

Finalmente, vale aclarar que la adquisición de actitudes prejuiciosas por parte de las personas se vincula con su proceso de socialización política. En ese sentido, las agencias de socialización (familia, escuela, grupo de pares) poseen un rol fundamental en la transmisión de actitudes prejuiciosas (Baron; Byrne, 2005). Por ello, a los fines de nuestro estudio interesó conocer el aporte predictivo del tipo de escuela secundaria a la cual asistieron los(as) participantes en su nivel de prejuicio hacia el colectivo social indagado.

Considerando estos antecedentes, se postularon las siguientes hipótesis:

- H1: SDO y RWA constituirán las dos variables con mayor poder predictivo (de signo positivo) sobre el nivel de prejuicio hacia trabajadoras sexuales en tanto grupo considerado disidente.

- H2: Las adhesiones a una religión y a una ideología política de derecha también ingresarán como variables predictoras (de signo positivo) de este tipo de prejuicio.

- $\mathrm{H}_{3}$ : El tipo de escuela secundaria a la cual asistieron los(as) participantes del estudio ingresará como variable predictora del nivel PTS.

\section{Metodología}

\section{Tipo de estudio}

Se realizó un estudio empírico cuantitativo transversal (Montero; León, 2007), el cual tuvo dos fases. En una primera fase, se procedió a la construcción y evaluación psicométrica de una escala de prejuicio hacia trabajadoras sexuales construida ad hoc para este estudio, mediante la realización de un estudio instrumental. En la segunda fase, se efectuó un estudio ex post facto retrospectivo simple de un grupo (Montero; León, 2007). 


\section{Fase 1. Estudio instrumental}

\section{Participantes}

Se trabajó con una muestra no probabilística accidental de $\mathrm{N}=245$ estudiantes de 17 a 60 años $(\mathrm{M}=20,98 ; \mathrm{DT}=6,7)$ del Ciclo de Nivelación ${ }^{1}$ de la Universidad Nacional de Córdoba, Argentina. De ellos(as), el 71 \% fueron mujeres y el $29 \%$, varones. Un $24,6 \%$ pertenecían a sectores socioeconómicos bajos; $58,7 \%$, a sectores medios; y $16,7 \%$, a nivel medio alto-alto.

\section{Procedimiento}

Se aplicó un cuestionario autoadministrado, previo consentimiento informado de los(as) participantes. La administración se realizó de manera colectiva en espacio áulico previa autorización del(la) docente a cargo, con una duración aproximada de 20 minutos. La información obtenida se analizó con el software estadístico SPSS 20.

\section{Instrumentos}

El cuestionario autoadministrado tenía la modalidad de cuestionario cerrado de alternativa fija, e incluía las siguientes escalas:

- Variables sociodemográficas: preguntas cerradas de alternativa fija para conocer el sexo, edad y nivel socioeconómico (en adelante NSE) de los(as) participantes.

- PTS: se creó una escala $a d$ hoc para este estudio. Los ítems fueron redactados considerando estudios previos vinculados al trabajo sexual en Argentina (Dreizik; Brussino, 2014; Dreizik; Roveres, 2013), así como investigaciones en torno a otros tipos de prejuicio (Cozzarelli; Wilkinson; Tagler, 2001; Henry; Hardin, 2006; Jarrell et al., 2014; Pettigrew; Meertens, 1995; Quillian, 1995). Se crearon 31 ítems que remiten a actitudes de prejuicio sutil (p. e.: "Me da lástima la situación en la que se encuentran las prostitutas/ trabajadoras sexuales"; "Las prostitutas/trabajadoras sexuales enseñan a sus hijos/as costumbres, maneras de ser y valores diferentes a los nuestros"; "Las prostitutas/trabajadoras sexuales son diferentes del resto de la sociedad en sus costumbres y formas de ser") y manifiesto (p. e.: "Me dan un poco de asco y rechazo las prostitutas/trabajadoras sexuales"; "La presencia de prostitutas/trabajadoras sexuales es una de las causas de la delincuencia y la violencia"; "Las prostitutas/trabajadoras sexuales son inmorales"). A su vez, y dado que el prejuicio constituye una actitud (Allport, 1954; Myers, 2007) los ítems incluidos se orientaron a la exploración de los tres componentes actitudinales: afectivo (p. e.: "Mis sentimientos hacia las prostitutas/trabajadoras sexuales

1. El Ciclo de Nivelación constituye el dispositivo de ingreso a la universidad pública en la Universidad Nacional de Córdoba. 
son generalmente positivos", item inverso), cognitivo ("Las prostitutas/trabajadoras sexuales son peligrosas") y conductual ("Debería restringirse el ingreso de prostitutas/ trabajadoras sexuales a ciertos establecimientos dado que siempre causan problemas”). De ellos, seis están redactados en sentido inverso (para una lectura más simple de los resultados, los puntajes de estos fueron invertidos antes de comenzar los análisis, de manera tal que vayan en el mismo sentido que los ítems positivos). Las opciones de respuesta son de tipo Likert de cinco anclajes, donde $1=$ totalmente en desacuerdo, $y$ $s=$ totalmente de acuerdo.

\section{Preparación de los datos}

Se evaluó el patrón de valores ausentes y los supuestos de normalidad. Ninguno de los ítems considerados superó niveles del $5 \%$ de casos perdidos, por lo cual se procedió a completar los valores perdidos con el método de sustitución de imputación por la media. Respecto de los supuestos de normalidad, se procedió a analizar los valores de curtosis y asimetría, considerando como criterio que valores entre $\pm 1,00$ son excelentes, y valores entre $\pm 2, \mathrm{oo}$, adecuados (George; Mallery, 2011). La escala de PTS propuesta registró 11 ítems con niveles excelentes de curtosis y asimetría; 18 con niveles adecuados y 2 con niveles no aceptables.

\section{Análisis de datos}

Se realizaron análisis factoriales exploratorios (AFE), usando el método de componentes principales, por ser uno de los más utilizados en este análisis (Pérez; Medrano, 2010). Se optó por una rotación oblicua promax, en función del grado de correlación entre los factores de la escala. Inicialmente, se comenzaron los análisis con todos los ítems administrados, incluyendo los cúrticos y asimétricos, dado que, según Garson (2011), el AFE es una prueba de correlación entre variables y no de significación, y por ende los supuestos de normalidad no son requisitos excluyentes. Para determinar el número de factores o dimensiones a extraer, se consideraron diversos criterios, siguiendo la recomendación de Pérez y Medrano (2010), quienes sostienen que utilizar un solo criterio puede subestimar o sobrestimar el número real de dimensiones de la escala considerada. Así, por una parte, se utilizó el criterio de autovalores iguales a 1 (Hair; Anderson; Tatham; Black, 1999; Tabachnick; Fidell, 2001), combinado con el análisis del gráfico de sedimentación. La aplicación de estos criterios estuvo supeditada a la adecuación de la estructura con la propuesta teórica que dio lugar a la construcción de la escala. Para dar cierre a esta primera fase instrumental, se analizó el nivel de confiabilidad de la escala mediante el índice Alpha de Cronbach, y se evaluó cómo se vería afectada la confiabilidad al eliminar cada uno de los ítems de la escala. 


\section{Fase 2. Estudio ex post facto}

\section{Participantes}

Se trabajó con estudiantes de grado de la Universidad Nacional de Córdoba y de la Universidad Católica de Córdoba, Argentina. Se decidió trabajar con ambas universidades, una pública estatal (laica) y una privada religiosa, a fines de aportar mayor variabilidad a la muestra. Se realizó un muestreo no probabilístico accidental de 381 estudiantes universitarios(as) de 17 a 60 años $(\mathrm{M}=20,08$; $\mathrm{DT}=5,63)$. El 64,3\% fueron mujeres y el restante $35,7 \%$, varones. Respecto del nivel socioeconómico, 16,3\% pertenecen a sectores bajos, $58,7 \%$ a sectores medios, y $24,9 \%$ a nivel medio alto-alto.

\section{Procedimiento}

Se aplicó un cuestionario autoadministrado, previo consentimiento informado de los(as) participantes. Al igual que en la fase anterior, se procedió a detallar los objetivos del estudio, y el carácter anónimo y confidencial de la participación en el mismo. La administración también se efectuó de forma colectiva en espacio áulico previa autorización del(la) docente a cargo, con una duración aproximada de 30 minutos. La información obtenida se procesó con el software estadístico SPSS 20.

\section{Instrumentos}

El cuestionario autoadministrado tenía la modalidad de cuestionario cerrado de alternativa fija, e incluía las siguientes escalas:

- Variables sociodemográficas: se evaluaron de igual forma que en la Fase 1.

- PTS: se consideró la versión de la escala obtenida tras los AFE realizados durante la fase instrumental. Así, se trabajó con una versión de 18 ítems (3 inversos) con formato de respuesta tipo Likert de 5 anclajes. La dimensión afectivo-conductual (9 ítems) evidenció un $\alpha=0,89$ y la dimensión cognitiva ( 9 ítems) un $\alpha=0,83$.

- Religión: se solicitó a los(as) participantes que indicaran si pertenecían a alguna religión (opción de respuesta Sí o No) y, en caso afirmativo, que especificaran a qué culto.

- Escuela secundaria: se requirió información en torno al tipo de escuela a la cual asistieron los(as) participantes durante el nivel medio. Las opciones de respuesta fueron: 1) escuela pública; 2) escuela privada religiosa; 3) escuela privada no religiosa.

- Orientación a la dominancia social (SDO): se incluyó la adaptación local de la Escala de SDO (Pratto et al., 1994), efectuada por Etchezahar, Prado-Gascó, Jaume y Brussino (2014). Esta versión posee diez ítems con opciones de respuesta en una escala Likert de 5 posiciones ( $1=$ totalmente en desacuerdo; $5=$ totalmente de acuerdo). Los ítems se 
agrupan en dos dimensiones, cada una de cinco ítems: oposición a la igualdad y orientación de dominancia grupal. Los índices de confiabilidad en el estudio de Etchezahar et al. (2014) fueron de $\alpha=0,74$ y $\alpha=0,85$, respectivamente. En este estudio, la escala mostró un $\alpha=0,62$ en su dimensión de orientación de dominancia grupal y $\alpha=0,76$ en la dimensión oposición a la igualdad.

- Autoritarismo del ala de derechas (RWA): se trabajó con la versión argentina de la escala de Right Wing Authoritarianism (Altemeyer, 1998; 2006), propuesta por Etchezahar (2012). Es una versión breve de la escala original que reúne seis ítems en una estructura unidimensional. Las opciones de respuesta se sitúan en una escala Likert de 5 posiciones (1=totalmente en desacuerdo; $5=$ totalmente de acuerdo). En el estudio de Etchezahar (2012), la escala presentó niveles de fiabilidad muy buenos $(\alpha=0,83)$; al igual que en nuestro estudio $(\alpha=0,81)$.

- Ideología política: se evaluó mediante una medida de autorreporte. Así, se solicitó a los(as) participantes que indicaran su autoposicionamiento ideológico en una escala que va de $1=$ totalmente de izquierda a $7=$ totalmente de derecha.

\section{Preparación de los datos}

Inicialmente, se evalúo el patrón de datos ausentes, ratificándose un porcentaje menor al 5 \%. Por ello, se procedió a completar la información faltante mediante el método de imputación por la media. Posteriormente, se analizaron los supuestos de normalidad.

\section{Análisis de datos}

Se efectuó un análisis de regresión múltiple con el método de pasos sucesivos en el marco del cual las dimensiones del PTS (afectivo-conductual y cognitiva) se incorporaron como variables dependientes; mientras que la edad, el nivel socioeconómico, la religión, la orientación de dominancia social (SDO), el autoritarismo de derechas (RWA) y la ideología política ingresaron como variables independientes.

\section{Resultados}

\section{Fase 1. Estudio instrumental}

El primer paso consistió en analizar la factibilidad de aplicación del análisis factorial a los datos en estudio, lo cual fue evaluado a través del índice de adecuación muestral KMO y la prueba de esfericidad de Bartlett. Respecto del primero, se obtuvo un valor de 0,914 ; mientras que la segunda arrojó resultados significativos $(\mathrm{gl}=465 ; \mathrm{p}<\mathrm{O}, \mathrm{OOO})$. Estos dos factores denotan la posibilidad de efectuar un AFE en nuestro caso. 
La primera solución factorial obtenida evidenció una estructura de siete factores que explican el $55 \%$ de la varianza conjunta. Por su parte, el gráfico de sedimentación sugiere la presencia de dos factores, lo cual resulta coincidente con la teoría que sirvió de base para la construcción de la escala que diferencia entre expresiones de prejuicio sutil, por un lado, y manifiesto, por el otro. En función de ello, se decidió repetir el análisis solicitando la extracción de dos factores. Los resultados consecuentes $(\mathrm{KMO}=0,914$; prueba de esfericidad de Barlettgl $=465 ; \mathrm{p}<0, \mathrm{Ooo})$ muestran una estructura bidimensional que explica el $35 \%$ de la varianza conjunta, en la cual los ítems 1 (La universidad debería tener un número de plazas especialmente dirigidas a las prostitutas/trabajadoras sexuales, para fomentar que accedan a estudios universitarios), 17 (No me molesta que las prostitutas/trabajadoras sexuales realicen la actividad pero deberían tener un lugar especial para hacerlo), 21 (Me da lástima la situación en la que se encuentran las prostitutas/trabajadoras sexuales) y 24 (Sería bueno que existan escuelas especiales para los(as) hijos(as) de las prostitutas/trabajadoras sexuales, más ajustadas a su marco de valores) no manifiestan saturación factorial en ninguna de las dos dimensiones. Por ende, se decide eliminarlos de a uno, de forma consecutiva, de los siguientes análisis.

Como resultado de estas eliminaciones progresivas, se obtiene una solución factorial de dos dimensiones $(\mathrm{KMO}=0,921$; prueba de esfericidad de Barlett $\mathrm{gl}=351$; p <0,ooo) que explica el 39,2\% de la varianza. Un análisis sobre el contenido teórico de los ítems de cada dimensión muestra que el primer factor (cuya varianza explicada es del $32,5 \%$ ) reúne de forma prioritaria ítems que remiten a aspectos afectivos y conductuales del prejuicio (a excepción de unos pocos), mientras que el segundo factor (varianza explicada de 6,7\%) agrupa, casi exclusivamente, ítems de carácter cognitivo (con excepción de uno conductual). Estos resultados sugieren que la lógica teórica de agrupación en dimensiones remite a aspectos asociados al carácter actitudinal del prejuicio hacia las trabajadoras sexuales, y no a la diferenciación entre expresiones sutiles o manifiestas del prejuicio. En consecuencia, se decide proceder eliminando de a uno el ítem de tipo conductual de la segunda dimensión factorial y los ítems de carácter cognitivo del primer factor. Los resultados $(\mathrm{KMO}=0,920$; prueba de esfericidad de Barlettgl $=253 ; \mathrm{p}<0,000$ ) muestran una solución bidimensional que explica el $41,5 \%$ de la varianza, con un factor compuesto de forma exclusiva por ítems de carácter cognitivo (segundo factor, varianza explicada de 7,8\%), y uno que reúne, principalmente, ítems que denotan aspectos afectivos y conductuales del prejuicio (primer factor, varianza explicada de 33,6\%).

La inspección de las cargas factoriales muestra que los ítems 3 (Las prostitutas/ trabajadoras sexuales suelen ser portadoras de infecciones de transmisión sexual), 5 (En Argentina muchas personas progresan de forma honesta. Las prostitutas/ 
trabajadoras sexuales podrían hacer lo mismo) y 13 (La presencia de prostitutas/ trabajadoras sexuales es una de las causas de la delincuencia y la violencia), que saturan en el segundo factor, evidencian una carga factorial baja (entre $0,335 \mathrm{y}$ o,466), por lo que se decide repetir el análisis sin estos ítems, lo cual redunda en un aumento de la varianza explicada de este factor. A su vez, tras estos análisis, se percibe que el ítem 27 (Debería restringirse el ingreso de prostitutas/trabajadoras sexuales a ciertos establecimientos dado que siempre causan problemas) es el que menor carga factorial $(0,369)$ muestra en la primera dimensión. Por ello, se lo retira y se repiten los análisis. De este modo, los resultados $(\mathrm{KMO}=0,927$; prueba de esfericidad de Barlett $\mathrm{gl}=171 ; \mathrm{p}<0,000)$ muestran una estructura de dos dimensiones que explica el $46,5 \%$ de la varianza ( $37,4 \%$ el primer factor, y $9,1 \%$ el segundo). Las cargas factoriales de los ítems son en todos los casos superiores a 0,50, excepto en el caso del ítem 25 (Las prostitutas/trabajadoras sexuales suelen ser drogadictas), que carga en el segundo factor $(0,469)$. Por ello, se decide eliminarlo y repetir el análisis.

Así, el modelo final obtenido es de carácter bidimensional, reúne 18 ítems ( 3 de los cuales son inversos) y explica el $48 \%$ de la varianza conjunta. El primer factor ( $38 \%$ de varianza explicada) está compuesto por 9 ítems que remiten a la dimensión afectivo-conductual del prejuicio; mientras que el segundo factor reúne 9 ítems (10\% de varianza explicada) que hacen referencia a la dimensión cognitiva del prejuicio hacia trabajadoras sexuales. Vale aclarar que los ítems retenidos dan cuenta, a su vez, de expresiones sutiles y manifiestas del PTS. En la Tabla 1 se puede apreciar la estructura final de la escala analizada.

Finalmente, los análisis de fiabilidad muestran que ambas dimensiones poseen un valor de Alpha de Cronbach muy bueno ( $\alpha=0,87$ la dimensión afectivo-conductual, $\mathrm{y} \alpha=0,83$ la dimensión cognitiva), que no aumenta tras la eliminación de ninguno de los componentes retenidos en la estructura final de la escala.

Esta estructura de la escala de prejuicio hacia trabajadoras sexuales es la que finalmente se utilizó para la realización de la Fase 2 del estudio.

\section{Fase 2. Análisis predictivo}

La segunda fase del estudio consistió en la identificación de las variables psicosociales (religión, orientación de dominancia social), psicopolíticas (autoritarismo del ala de derechas, ideología política) y sociodemográficas (edad, sexo, nivel educativo, nivel socioeconómico) que colaboran en la predicción del prejuicio hacia trabajadoras sexuales (PTS). Se consideró como variable dependiente a ambas dimensiones del PTS (afectivo-conductual y cognitiva). 
TABLA 1 Dimensiones del prejuicio hacia trabajadoras sexuales

Prejuicio hacia trabajadoras sexuales

Componente

\section{Afectivo- \\ conductual \\ Cognitivo}

Ítem 19. No me gustaría sentarme al lado de una prostituta/ trabajadora sexual en el colectivo (contenido manifiesto)

Ítem 22. Tengo sentimientos negativos hacia las prostitutas/ trabajadoras sexuales (contenido manifiesto)

0,835

Ítem 23. Me molestaría tener como vecina a una prostituta/ trabajadora sexual (contenido manifiesto)

Ítem 26. Me dan un poco de asco y rechazo las prostitutas/ trabajadoras sexuales (contenido manifiesto)

Ítem 7. Las prostitutas/trabajadoras sexuales me hacen sentir incómodo/a (contenido manifiesto)

0,706

Ítem 9. Trato de evitar relacionarme con prostitutas/

trabajadoras sexuales (contenido manifiesto)

0,624

Ítem 15. Tendría como amiga a una prostituta/trabajadora sexual $^{*}$ (contenido manifiesto)

0,599

Ítem 4. Me desagradan las prostitutas/trabajadoras sexuales (contenido manifiesto)

Ítem 2. Mis sentimientos hacia las prostitutas/trabajadoras sexuales son generalmente positivos* (contenido sutil)

Ítem 29. No creo que haya muchas diferencias en las creencias e ideas de las prostitutas/trabajadoras sexuales y las de otras mujeres ${ }^{*}$ (contenido sutil)

Item 6. Creo que los valores religiosos y éticos de las prostitutas/trabajadoras sexuales son diferentes a los nuestros (contenido sutil)

Ítem 14. Las prostitutas/trabajadoras sexuales son inmorales (contenido manifiesto)

Ítem 8. Las prostitutas/trabajadoras sexuales enseñan a sus hijos/as costumbres, maneras de ser y valores diferentes a los nuestros (contenido sutil)

Ítem 30. Las prostitutas/trabajadoras sexuales son como cualquier otro/a trabajador/a* (contenido sutil)

Ítem 11. Las prostitutas/trabajadoras sexuales buscan ganar la plata fácil (contenido manifiesto)

0,711

Ítem 28. Las prostitutas/trabajadoras sexuales son diferentes del resto de la sociedad en sus costumbres y formas de ser (contenido sutil) 


\begin{tabular}{|c|c|}
\hline $\begin{array}{l}\text { Ítem 10. Las prostitutas/trabajadoras sexuales son una amenaza } \\
\text { para las familias y la sociedad (contenido manifiesto) }\end{array}$ & 0,554 \\
\hline \multirow[t]{2}{*}{$\begin{array}{l}\text { Ítem 18. Las prostitutas/trabajadoras sexuales son un mal } \\
\text { ejemplo para los niños (contenido manifiesto) }\end{array}$} & 0,502 \\
\hline & $\alpha=\mathbf{0}, \mathbf{8 3}$ \\
\hline * Ítems inversos. & \\
\hline
\end{tabular}

En primer lugar, y para los fines de exploración inicial de los datos, se efectuaron correlaciones bivariadas entre ambas dimensiones del PTS entre sí, y entre las variables independientes del estudio con ambas dimensiones de la variable dependiente PTS. Respecto del primer punto, se ratificó una relación positiva significativa y alta $(0,688)$ entre las dos dimensiones actitudinales del PTS consideradas, denotando que se trata de aspectos vinculados de un mismo fenómeno. En lo que concierne a la vinculación entre las dimensiones del PTS con las variables independientes del estudio, se observó una correlación inversa baja, pero significativa, entre la edad de los(as) participantes y su nivel de PTS de tipo afectivo-conductual, esto es, a más edad menor nivel de este tipo de prejuicio. A su vez, se destaca una asociación positiva, moderada y significativa entre el hecho de adherir a algún culto religioso y el nivel de PTS en ambas dimensiones, es decir, las personas que se consideran religiosas manifiestan más prejuicio hacia esta población que quienes no poseen religión. En otro orden, ambas dimensiones del PTS muestran una relación moderada, positiva y significativa con el autoposicionamiento ideológico, de forma tal que son las personas que más adhieren a posiciones de derecha las que muestran niveles más altos de PTS, tanto cognitivo como afectivo-conductual. Por otra parte, ambas dimensiones de la orientación a la dominancia social manifiestan una relación positiva y significativa con las dos dimensiones del PTS consideradas, aunque esta relación es más fuerte en el caso de la orientación a la dominancia grupal que en el caso de la oposición a la igualdad. Por último, el autoritarismo del ala de derechas muestra una correlación positiva y significativa con ambas dimensiones del PTS, la cual es moderada para el caso de la dimensión afectivo-conductual y alta para la dimensión cognitiva (ver Tabla 2).

Tras esta exploración inicial de los datos, se procedió a la realización del análisis de regresión múltiple con el método de pasos sucesivos, incluyendo las variables que mostraron relaciones significativas con la variable dependiente del estudio. En la Tabla 3 y la Tabla 4 pueden observarse los modelos finales obtenidos para ambas dimensiones del PTS analizadas. 
TABLA 2 Relación de las dimensiones del PTS con las variables independientes del estudio

Variables independientes

Dimensiones del PTS

\begin{tabular}{|c|c|c|}
\hline & $\begin{array}{l}\text { Afectivo- } \\
\text { conductual }\end{array}$ & Cognitivo \\
\hline Nivel socioeconómico & 0,044 & $-0,004$ \\
\hline Edad & $-0,141^{* *}$ & $-0,033$ \\
\hline Sexo & 0,267 & 0,310 \\
\hline Religión & $0,370^{*}$ & $0,366^{*}$ \\
\hline Escuela secundaria & 0,315 & 0,298 \\
\hline Autoposicionamiento ideológico & $0,304^{* *}$ & $0,294^{* *}$ \\
\hline Orientación de dominancia grupal (SDO) & $0,291^{* *}$ & $0,319^{* *}$ \\
\hline Oposición a la igualdad (SDO) & $0,188^{* *}$ & $0,166^{* *}$ \\
\hline Autoritarismo del ala de derechas (RWA) & $0,358^{* *}$ & $0,433^{* *}$ \\
\hline
\end{tabular}

Tal como se observa en la Tabla 3, el autoritarismo del ala de derechas y la oposición a la igualdad (SDO) son las variables que más aportan a la predicción de la dimensión afectivo-conductual del prejuicio hacia trabajadoras sexuales. En ambos casos, se trata de una influencia positiva, indicando que, a mayor autoritarismo y mayor oposición a la igualdad, mayor nivel de prejuicio. Por su parte, el hecho de pertenecer a una religión (versus quienes manifiestan no hacerlo) también colabora en la predicción de esta dimensión de PTS. Al mismo tiempo, la adhesión a una ideología política de derecha ingresa en el modelo predictivo de forma positiva, mostrando que las personas más conservadoras son también quienes manifiestan niveles más altos de PTS afectivo-conductual. Vale destacar que estas variables explican un $24 \%$ de la varianza de la dimensión afectivo-conductual del prejuicio hacia trabajadoras sexuales $\left(\mathrm{R}^{2}=0,240\right)$.

En lo que concierne a la dimensión cognitiva del prejuicio, es nuevamente el autoritarismo del ala de derechas la variable que mayor peso predictivo manifiesta. Específicamente, mientras mayores niveles de este autoritarismo manifieste una persona, se observarían mayores niveles de PTS en su dimensión cognitiva. Le siguen en su aporte predictivo la oposición a la igualdad (dimensión de SDO) y la religión, ambos con influencia positiva. Hasta aquí se trata de las mismas variables 


\begin{tabular}{|l|l|}
\hline TABLA 3 & $\begin{array}{l}\text { Influencia de las variables independientes sobre el nivel de prejuicio } \\
\text { hacia trabajadoras sexuales de los(as) participantes (dimensión } \\
\text { afectivo-conductual) }\end{array}$ \\
\hline
\end{tabular}

\begin{tabular}{llccc} 
& \multicolumn{3}{c}{ PTS - Dimensión afectivo-conductual } \\
\hline & $\mathbf{r}$ & $\boldsymbol{\beta}$ & $\mathbf{S i g}$ \\
\hline Autoritarismo del ala de derechas & $0,359^{* *}$ & 0,290 & 0,000 \\
\hline Oposición a la igualdad (SDO) & $0,193^{* *}$ & 0,204 & 0,000 \\
\hline Religión & $0,267^{* *}$ & 0,192 & 0,000 \\
\hline Autoposicionamiento ideológico & & $0,298^{* *}$ & 0,137 & 0,006 \\
\hline & $\mathbf{R}$ & & $\mathbf{0 , 4 8 6}$ & \\
\hline & $\mathbf{R}^{2}$ & & $\mathbf{0 , 2 4 0}$ & \\
\hline
\end{tabular}

TABLA 4

Influencia de las variables independientes sobre el nivel de prejuicio hacia trabajadoras sexuales de los(as) participantes (dimensión cognitiva)

\begin{tabular}{|c|c|c|c|}
\hline & \multicolumn{3}{|c|}{ PTS - Dimensión cognitiva } \\
\hline & $\mathbf{r}$ & $\boldsymbol{\beta}$ & Sig \\
\hline Autoritarismo del ala de derechas & $0,441^{* *}$ & 0,371 & 0,000 \\
\hline Oposición a la igualdad (SDO) & $0,159^{* *}$ & 0,155 & 0,004 \\
\hline Religión & $0,263^{* *}$ & 0,180 & 0,000 \\
\hline \multirow[t]{3}{*}{ Orientación de dominancia grupal (SDO) } & $0,312^{* *}$ & 0,136 & 0,022 \\
\hline & & $\mathbf{0 , 5 2 7}$ & \\
\hline & & $\mathbf{0 , 2 8 0}$ & \\
\hline
\end{tabular}

que ingresaron en el modelo anterior. No obstante, de forma diferencial, en este modelo la orientación de dominancia grupal (SDO) también aporta peso predictivo al modelo, mientras que el autoposicionamiento ideológico no ingresó en el mismo. Estas variables explican casi un $30 \%$ de la varianza del PTS $\left(\mathrm{R}^{2}=0,280\right)$. 


\section{Discusiones}

Un primer objetivo del presente trabajo giró en torno a la construcción y validación de una escala orientada a explorar el nivel de prejuicio que las personas manifiestan hacia las trabajadoras sexuales, colectivo de interés de nuestro estudio. La escala construida mostró adecuadas propiedades psicométricas, por lo cual puede ser utilizada para conocer este aspecto en el ámbito local. Ello constituye un aporte de relevancia en tanto la bibliografía indica, por una parte, que la mayor parte de las indagaciones sobre el prejuicio se han efectuado respecto del racial, descuidándose otras poblaciones, como la que indagamos aquí (Quiles del Castillo et al., 2003). En segundo término, la concepción del prejuicio como generalizado también ha impactado en una carencia de instrumentos que midan, de manera específica, el prejuicio hacia ciertos grupos. En el caso de las trabajadoras sexuales, en el mejor de los casos son incluidas en las investigaciones sobre grupos disidentes, pero no se contaba, hasta la actualidad, con una escala que de forma específica explore el nivel de prejuicio hacia este colectivo.

Por otra parte, los análisis predictivos permitieron identificar algunas de las variables psicosociales y psicopolíticas que posibilitarían evidenciar la presencia de este tipo de prejuicio en la población. Nuestros hallazgos han corroborado las hipótesis postuladas al inicio del trabajo, y construidas sobre la base de antecedentes empíricos previos, excepto en lo que concierne a la influencia de la escuela como agencia de socialización política. Específicamente, al concebirse a las trabajadoras sexuales como parte de los grupos disidentes, esto es, colectivos sociales que no solo se perciben como amenazantes del orden y las tradiciones, sino también en pie de disputa por recursos materiales y simbólicos concretos (Etchezahar et al., 2017; Sibley; Duckitt, 2008), tanto RWA como SDO ingresan en los modelos con aportes de signo positivo.

Aun así, vale destacar que es el autoritarismo del ala de derechas la variable que más aporta a la predicción en ambas dimensiones del PTS exploradas, al tiempo que, en lo concerniente a SDO, solo en la dimensión cognitiva ingresan ambas dimensiones (oposición a la igualdad y orientación de dominancia grupal). Ello podría estar sugiriendo que, a pesar de ser considerado un colectivo en disputa por recursos, es la connotación moral del autoritarismo del ala de derechas la que tiene mayor peso en la población en estudio. Es decir, el sentimiento de amenaza de las tradiciones y las costumbres parece ser más relevante para los(as) estudiantes encuestados(as), al momento de expresar su nivel de prejuicio hacia las trabajadoras sexuales. Este hallazgo podría también vincularse con el hecho de que la religión ingresó en ambos modelos como variable predictora del nivel de PTS de los(as) participantes, sugiriendo cierta matriz conservadora y moralista que da sostén a este tipo de actitudes prejuiciosas. 
En otro orden, la ideología política solo ingresó como variable predictora en el modelo específico de prejuicio de tipo afectivo-conductual. Ello quizás indique una mayor ligazón de las personas de derecha con manifestaciones emocionales del prejuicio hacia este colectivo, entre los que se cuentan sentimientos tales como incomodidad, asco y rechazo. Finalmente, cabe puntualizar que ninguna de las variables sociodemográficas indagadas (sexo, edad, nivel socioeconómico) efectuó un aporte a los modelos predictivos. Si bien se trata de un grupo bastante homogéneo y una muestra con un $\mathrm{N}$ modesto, lo cual constituye una de las limitaciones más relevantes del presente estudio, este hallazgo puede dar cuenta de la mayor relevancia de las variables psicosociales y psicopolíticas para la comprensión de este tipo de fenómenos sociales. No obstante, futuros estudios con población general podrían colaborar en la elucidación de este aspecto.

\section{Referencias}

Allport, Gordon (1954). The Nature of Prejudice. Reading, MA: Addition-Wesley.

Altemeyer, Bob (1998). The Other "Authoritarian Personality". En Advances in Experimental Social Psychology (Vol.30) (pp. 47-92), editado por Mark P. Zanna. Orlando, FL: Academic Press. https://doi.org/10.1016/soo65-2601(o8)60382-2

Altemeyer, Bob (2006). The Authoritarians. Winnipeg: University of Manitoba.

Baron, Robert; Byrne, Donn (2005). Psicología social (10. ${ }^{\mathrm{a}}$ ed.). México: Pearson Prentice Hall.

Civalero, Luciana; Brussino, Silvina (2016). Análisis psicosocialy psicopolítico del prejuicio hacia inmigrantes en estudiantes terciariosy universitarios de Buenos Aires, Córdoba, Saltay Neuquén (Tesis de pregrado). Universidad Nacional de Córdoba, Córdoba, Argentina.

Cozzarelli, Catherine; Wilkinson, Anna V.; Tagler, Michael J. (2001). Attitudes toward the Poor and Attributions for Poverty. Journal of Social Issues, 57, 207-227.

Cunningham, Jean; Dollinger, Stephen; Satz, Madelyn; Rotter, Nancy (1991). Personality Correlates of Prejudice Against AIDS Victims. Bulletin of the Psychonomic Society, 29, 165-167. https://doi.org/10.3758/bfo3335225

Dreizik, Matías; Brussino, Silvina (agosto, 2014). Traspasando la retórica de lo privado para construir la identidad política en torno al trabajo. Trabajo presentado en el $2 .^{\circ}$ Congreso Iberolatinoamericano de Psicología Política, Asociación Ibero-Latinoamericana de Psicología Política y Universidad Autónoma de México, México D. F., México. 
Dreizik, Matías; Roveres, Fabricio (2013). Trabajadoras sexuales y agremiación: protectores que brinda la organización AMMAR ante los riesgos psicosociales. Psicología, Conocimiento y Sociedad,3(1), 64-92. Recuperado de https://revista.psico.edu.uy/index.php/revpsicologia/ article/view/72/114

Duckitt, John (2006). Differential Effects of Right Wing Authoritarianism and Social Dominance Orientation on Outgroup Attitudes and Their Mediation by Threat from and Competitiveness to Outgroups. Personality and Social Psychology Bulletin, 32, 684-696. https:// doi.org/10.1177/0146167205284282

Duckitt, John; Sibley, Chris G. (2007) Right Wing Authoritarianism, Social Dominance Orientation and the Dimensions of Generalized Prejudice. European Journal of Personality Eur. J. Pers., 21, 113-13o. https://doi.org/10.1002/per.614

Duckitt, John; Wagner, Claire; Du Plessis, Ilouize; Birum, Ingrid (2002). The Psychological Bases of Ideology and Prejudice: Testing a Dual Process Model. Journal of Personality and Social Psychology, 83, 75-93. https://doi.org/10.1037/oo22-3514.83.1.75

Etchezahar, Edgardo (2012). Las dimensiones del autoritarismo: análisis de la escala de autoritarismo del ala de derechas (RWA) en una muestra de estudiantes universitarios de la Ciudad de Buenos Aires. Revista Psicología Política, 12(25), 591-603.

Etchezahar, Edgardo; Prado-Gascó, Vicente; Jaume, Luis; Brussino, Silvina (2014). Validación argentina de la Escala de Orientación a la Dominancia Social. Revista Latinoamericana de Psicología, 46(1), 35-43.

Etchezahar, Edgardo; Ungaretti, Joaquín; Brussino, Silvina (mayo, 2015). Autoritarismo, dominancia e ideología política en el prejuicio hacia inmigrantes bolivianos. Trabajo presentado en I Congreso Nacional de Psicología, Universidad Nacional de San Luis, San Luis, Argentina.

Etchezahar, Edgardo; Ungaretti, Joaquín; Rabbia, Hugo Hernán (2017). ¿Por qué nos cuesta tanto vivir juntos/as? Una mirada psico-política del prejuicio, los estereotipos y la discriminación. En Políticamente (pp. 209-230), coordinado por Silvina Brussino. Buenos Aires: CONICET. Recuperado de https://rdu.unc.edu.ar/handle/11086/4910

Fonseca-Hernández, Carlos (septiembre, 2004). Mujeres migrantes latinoamericanas en el trabajo sexual en Barcelona: grupo de discusión sobre el poder y el sufrimiento de las mujeres en la industria del sexo. Trabajo presentado en el I Congresso da Associação Latino Americana de População, ALAP, Caxambú, Brasil.

Garson, David (2011). Factor Analysis. Recuperado de http://faculty.chass.ncsu.edu/garson/ PA765/factor.htm\#normality

Gatica, Lucas; Martini, Juan Pablo; Dreizik, Matías; Imhoff, Débora (2017). Predictores psico-sociales y psico-políticos de la justificación de la desigualdad social. Revista de Psicología, 35(1), 279-310. https://doi.org/10.18800/psico.201701.010 
George, Darren; Mallery, Paul (2011). SPSS for Windows Step by Step: A Simple Guide and Reference 18.o Update (11. ${ }^{\mathrm{a}}$ ed.). Boston, MA: Allyn \& Bacon.

Hair, Joseph F.; Anderson, Rolph E.; Tatham, Ronald L.; Black, William C. (1999). Análisis multivariante. Madrid: Prentice Hall Iberia.

Henry, P.J.; Hardin, Curtis D. (2006). The Contact Hypothesis Revisited: Status Bias in the Reduction of Implicit Prejudice in the United States and Lebanon. Psychological Science, 17(10), 862-868. https://doi.org/10.1111/j.1467-9280.2006.01795.x

Jarrell, Kay; Ozymy, Joshua; Gallagher, John; Hagler, Debra; Corral, Camille; Hagler, Andrew (2014). Constructing the Foundations for Compassionate Care: How Service-learning Affects Nursing Students' Attitudes towards the Poor. Nurse Education in Practice, 14, 299-303.

Juliano, Dolores (2003). Prostitución: el modelo de control sexual femenino a partir de sus límites. En Sociología de la sexualidad (pp. 178-197), editado por Raquel Osborne; Oscar Guash. Madrid: Centro de Investigaciones Sociológicas/Siglo XXI.

Juliano, Dolores (2005). El trabajo sexual en la mira - Polémicas y estereotipos. Cadernos Pagu, $25,79-106$.

Montero, Ignacio; León, Orfelio (2007). Guía para nombrar los estudios de investigación en Psicología. International Journal of Clinical and Health Psychology, 3, 847-862.

Musto, Clara; Trajtenberg, Nico (2011). Prostitución y trabajo sexual: el estado de arte de la investigación en Uruguay. Revista de Ciencias Sociales, 24(29), 138-156. Recuperado de https://dialnet.unirioja.es/servlet/articulo?codigo=3963214

Myers, David (2007). Psicología social. México: McGraw Hill.

Norton, Aaron; Herek, Gregory (2013). Heterosexuals' Attitudes toward Transgender People: Findings from a National Probability Sample of US Adults. Sex Roles, 68(11-12), 738-753. https://doi.org/10.1007/s11199-011-0110-6

Páez, José; Hevia, Guillermo; Pesci, Florencia; Rabbia, Hugo (2015) Construcción y validación de una escala de actitudes negativas hacia personas trans. Revista de Psicología, 33(1), 151-188.

Pecheny, Mario; Petracci, Mónica (2006). Derechos humanos y sexualidad en la Argentina. Horizontes Antropológicos, 12 (26), 43-69.

Pérez, Edgardo; Medrano, Leonardo (2010). Análisis factorial exploratorio: bases conceptuales y metodológicas. Revista Argentina de Ciencias del Comportamiento, 2(1), 58-66.

Pettigrew, Thomas; Meertens, Roel (1995). Subtle and Blatant Prejudice in Western Europe. European Journal of Social Psychology, 25, 57-75. http://dx.doi.org/10.1002/ejsp.2420250106 
Pratto, Felicia, Sidanius, Jim; Stallworth, Lisa M.; Malle, Bertram F. (1994). Social Dominance Orientation: A Personality Variable Predicting Social and Political Attitudes. Journal of Personality and Social Psychology, 67(4), 741-763. http://dx.doi.org/10.1037/0022-3514.67.4.741

Quiles del Castillo, Nieves; Betancor-Rodríguez, Verónica; Rodríguez-Torres, Ramón; Rodríguez-Pérez, Armando; Coello-Martel, Efrén (2003). La medida de la homofobia manifiesta y sutil. Psicothema, 15(2), 197-204. Recuperado de http://www.psicothema.com/ psicothema.asp?id=1045

Quillian, Lincoln (1995). Prejudice as a Response to Perceived Group Threat: Population Composition and Anti-immigrant and Racial Prejudice in Europe. American Sociological Review, 6o(4), 586-611.

Rottenbacher, Jan Marc; Espinosa, Agustín; Magallanes, José Manuel (2011). Analizando el prejuicio: bases ideológicas del racismo, el sexismo y la homofobia en una muestra de habitantes de la ciudad de Lima. Revista Psicología Política, 11(22), 225-246. Recuperado de https://dialnet.unirioja.es/servlet/articulo?codigo $=3898832$

Roveres, Fabricio; Dreizik; Matías; Correa, Ana (2007). Contenidos y significados respecto del presente y futuro de mujeres nucleadas en AMMAR, en situación de trabajo sexual (Tesis de pregrado). Universidad Nacional de Córdoba, Córdoba, Argentina.

Sibley, Chris; Duckitt, John (2008). Personality and Prejudice: A Meta-analysis and Theoretical Review. Personality and Social Psychology Review, 12, 248-279. https://doi. org/10.1177/1088868308319226

Tabachnick, Barbara \& Fidell, Linda (2001). Using Multivariate Statistics. New York: Harper \& Row.

Ungaretti, Joaquín; Etchezahar, Edgardo; Brussino, Silvina (2015). La percepción de peligro y competencia como factores predictores del prejuicio hacia diferentes grupos sociales. Escritos de Psicología, 8(1), 30-37. https://doi.org/10.5231/psy.writ.2015.0912 